\title{
Simulation-Based Otorhinolaryngology Emergencies Boot Camp: Part 3: Complex Teamwork Scenarios and Conclusions
}

\author{
Ellen S. Deutsch, MD, FACS; Kelly M. Malloy, MD, FACS; Sonya Malekzadeh, MD, FACS
}

Key Words: Simulation, boot camp, resident education, cricothyrotomy, laryngoscopy, bronchoscopy, epistaxis, emergency, medical education, surgical education.

Laryngoscope, 124:1570-1572, 2014

\section{INTRODUCTION}

This is the final article in a series describing the Otorhinolaryngology (ORL) Emergencies Boot Camp. Previous articles covered curriculum design and basic to more advanced emergency skills. This article describes how the course culminates in two learning stations addressing more complicated patient scenarios that also require judgment, communication, and teamwork in addition to technical skills.

\section{COMPLEX SCENARIOS}

Complex scenarios simulate a clinical situation that learners must manage as a team, effectively putting together the skills and knowledge they accumulated earlier in the boot camp. In addition to practicing procedures, complex scenarios also allow exploration of team training, communication skills, situational awareness, managing hierarchies, and establishing leadership. ${ }^{1-3}$ We have found the following cases to be particularly effective in allowing junior ORL learners to coalesce and implement newly acquired skills: 1) "Cannot intubate,

From the Department of Anesthesia and Critical Care (E.S.D.), The Children's Hospital of Philadelphia, Philadelphia, Pennsylvania; Department of Otolaryngology-Head and Neck Surgery (K.M.м.), University of Michigan Health System, Ann Arbor, Michigan; and the Department of Otolaryngology-Head and Neck Surgery (S.M.), MedStar Georgetown University Hospital, Washington, DC, U.S.A.

Additional Supporting Information may be found in the online version of this article.

Editor's Note: This Manuscript was accepted for publication November 12, 2013.

The course was conducted at Simulation \& Training Environment Laboratory and MedStar Georgetown University Hospital.

The authors have no funding, financial relationships, or conflicts of interest to disclose.

Send correspondence to Kelly M. Malloy, MD, Department of Otolaryngology-Head and Neck Surgery, University of Michigan Health System, 1500 E. Medical Center Drive, First Floor, Taubman Center, Ann Arbor, MI 48109. E-mail: kellymal@umich.edu

DOI: 10.1002/lary.24570 cannot ventilate" precipitated by angioedema; and 2) Neck hematoma following thyroid surgery. Other scenarios may be appropriate, and creativity is encouraged in developing personal scenarios.

Following an orientation to the manikin's capabilities, the stem circumstances of the simulation are shared with the residents (e.g., "You are called to the emergency department [ED] to see a 42-year-old Asian male 6 hours after an uneventful thyroidectomy because the patient has returned with a complaint of neck swelling."). As diagnosis is a component of the learning objectives, the medical condition of the manikin and the expected actions of the care providers are not provided. The learning objectives and relevant articles are provided to faculty in advance (Supplemental Information 1).

Full-sized adult, child, and infant high-technology manikins are commercially available; we have used predominantly the SimMan 3G (Laerdal, Wappingers Falls, NY). High-technology manikins allow programming the "patient" to display appropriate physical characteristics and vital signs that respond according to interventions (or lack thereof). The manikins can demonstrate laryngospasm, stridor, tongue and pharyngeal edema, and trismus. It is possible to perform a cricothyroidotomy on certain manikins. A neck hematoma is created by placing a blood pack underneath disposable neck skin (Fig. 1). A faculty member provides the voice for the manikin and answers questions residents may ask the patient directly. The scene needs to be staged appropriately to encourage the suspension of disbelief and maintain a high-fidelity experience. As our scenarios take place in the ED, the manikins lie in hospital beds and wear hospital gowns. A cart with equipment found in a typical ED is provided, including oxygen nasal cannula, intubating laryngoscopes, and simple surgical equipment such as disposable scalpels. Items that are not normally found in an average ED, such as a nasopharyngolaryngoscopes (NPLs), are absent; if requested, it may take some time for them to arrive. Equipment and supplies are listed in Supplemental Information 2. 

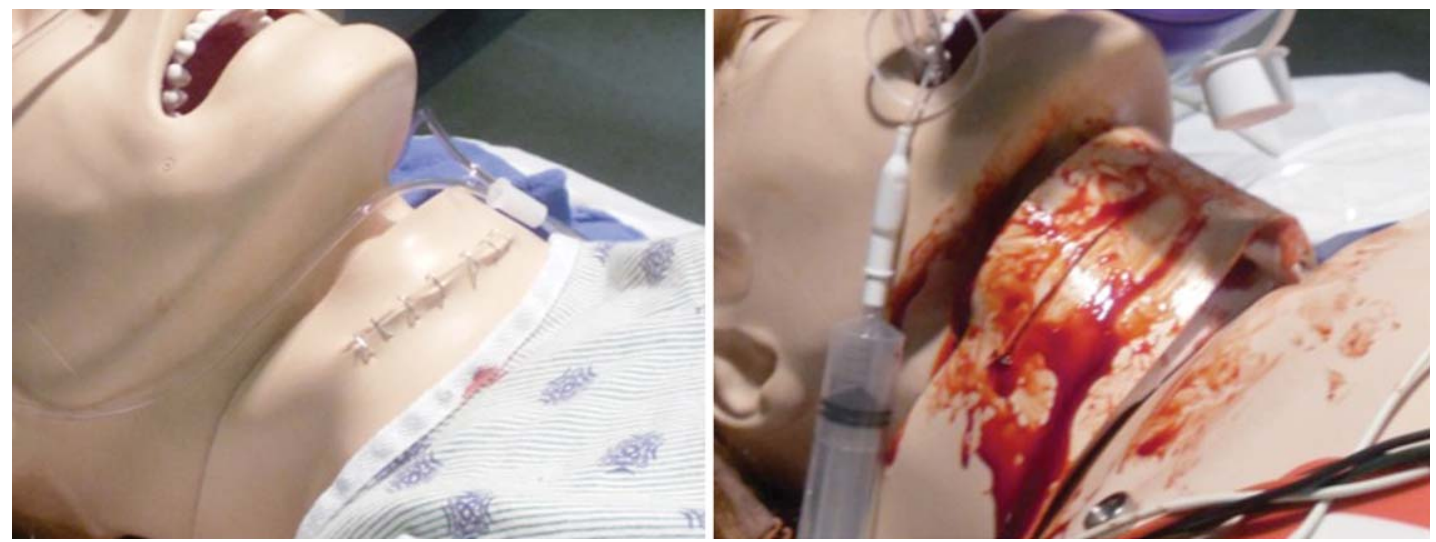

Fig. 1. Photo demonstrating the appearance of the manikin with a postoperative hematoma before and after opening of the wound with evacuation of the clot.

"Confederates," faculty, or simulation center staff who role play as ED nurses, attendings, an ORL attending on the phone, or family members can provide information to the learners to facilitate the simulation.

\section{Scenario Details: Angioedema Cannot Intubate, Cannot Ventilate}

The manikin is programmed to exhibit stridor, tongue edema, and poor oxygenation on room air. Upon the residents' arrival, the ED attending informs them that the patient has lip edema and garbled speech. A history of hypertension and angiotensin-converting enzyme inhibitor use is provided only if requested by the residents. The residents can ask the patient questions, and a confederate faculty member answers (using a "hot potato" voice) via microphone. After a few minutes, the patient communicates that breathing is becoming difficult and then loses consciousness as the oxygen saturations drop. Learners should start to intervene early in the case, applying supplemental oxygen and attempting to place a nasal airway or other airway manipulation. If they ask for an NPL, it is not readily available, and the patient will decompensate before it arrives. Once the patient is unconscious, the learners should attempt to ventilate, and when finding this impossible, attempt to intubate. By this time, the manikin cannot be intubated because of tongue and pharyngeal edema, neck rigidity, and trismus. The learners should proceed to surgical cricothyroidotomy as the only remaining option to secure the patient's airway. Once an endotracheal tube is placed in the surgical airway and good tidal volumes delivered by bag ventilation, the patient's oxygen saturation recovers. The scenario is complete when the residents express the next step for their plan (i.e., conversion of the cricothyroidotomy to tracheotomy within 24 hours).

Usually, two residents work together to care for this patient; one assumes the role of a more senior resident. The faculty guides a debriefing of the scenario immediately following completion of the exercise, which addresses communication and teamwork as well as technical skills.

\section{Scenario Details: Acute Hematoma Following Thyroid Surgery}

In this scenario, the manikin is prepared with a blood-soaked gauze dressing over a closed lateral neck incision and a blood pack inserted under the disposable neck skin causing obvious neck fullness. The patient presents to the ED having had thyroid surgery earlier in the day and reports noticing increased swelling and pain in the anterior neck. The manikin is slightly tachycardic, but oxygenation saturations are normal at this point; the manikin also exhibits stridor. A faculty member speaking for the patient expresses anxiety and a feeling that it is becoming difficult to breathe. The learners have time to ask questions and formulate a plan that often includes inserting a nasal airway and providing supplemental oxygen, and request an NPL for airway evaluation. If the hematoma is diagnosed, the wound opened, and the collection drained, the patient then feels better and the stridor resolves. Most junior ORL residents are hesitant to drain the hematoma initially; the manikin's condition will continue to deteriorate until they perform this intervention. The patient's oxygen saturation drops and the patient becomes less responsive. The manikin is programmed to prevent intubation as well as ventilation, with pharyngeal edema, trismus, and laryngospasm. If sedatives or paralytics are administered, the patient's condition worsens. Drainage of the hematoma, by removing the dressing and skin staples, and bluntly puncturing into the hematoma, allows the airway to improve (i.e., all obstructions are turned off), and the learners can then intubate safely. The scenario ends when the residents have devised a plan for going back to the operating room to explore the wound and control bleeding. Debriefing addresses decision making, including discussion about sedatives and paralytic agents, technical skills, and teamwork and communication. Variations include incorporating an obstructionist ED attending (confederate) who is reticent to let the learners open the wound without the surgical attending being present. This generates significant discussion about the nuances of resident attending communication and attending expectations. 


\section{IT'S 3 AM. . DO I REALLY NEED TO CALL MY ATTENDING?}

The boot camp experience is intended to help residents develop the skills and judgment necessary to manage emergencies. For certain situations, residents need to contact their faculty, but residents, particularly new ones, may be reluctant to do this.

Cases of common urgent ED consults, uncommon but critical emergencies, and typical inquiries that are not emergent are presented to a group of learners via a slide presentation (Fig. 2). To make appropriate decisions and intervene correctly, residents discuss what questions to ask and when to request assistance. The audience response system keypad allows them to respond in an anonymous fashion. The residents' answers, in aggregate,

\section{You are called to the ED to see this aural foreign body. It's still alive. What is the best option?}
(a) Instill lidocaine
Instill mineral oil
Instill alcohol
Suction it out

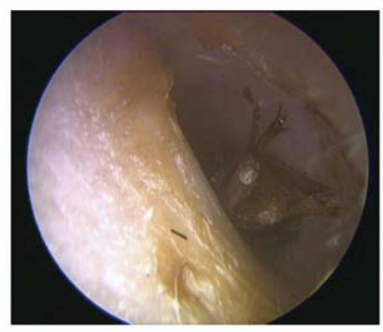

\section{Do you really want to call your Attending?}

(b)
Yes, call Attending
No, wait until morning

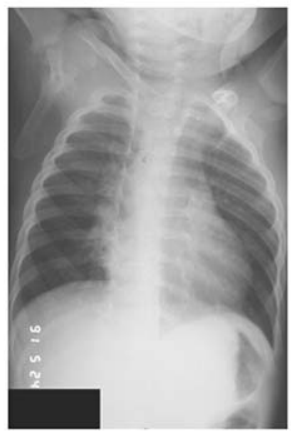

Fig. 2. Screen shots from audience response system slide presentation demonstrating clinical examples of (A) aural foreign body (insect) and (B) aspiration of foreign body (demonstrated by hyperinflation of right lung in right lateral decubitus position). Additional questions include when to call the attending and what the next best step in management would be. ED = emergency department. are demonstrated immediately, which provides the faculty with information about the knowledge of the residents as a group. Several faculty members participate and respond to the residents' selections as a panel; they can discuss initial management, address incorrect answers, and reinforce correct decisions. Subsequent slides provide followup questions, additional information, or explanations. The residents hear multiple perspectives, or they may learn that there is general agreement about certain principles or conditions.

\section{DISCUSSION}

Each of the ORL Emergency Boot Camp simulations, ranging from basic tasks to complex scenarios, could be set up as an isolated learning event and inserted into a more traditional curricular format. Some faculty adapt individual simulation stations and substitute them for 1-hour lectures for their core educational programs. However, the boot camp experience is much more than just the sum of its parts. The opportunity for residents to collaborate with residents from other institutions, and to receive individualized instruction and feedback from faculty from a range of institutions, is unique and invaluable. Perhaps most importantly, the residents learn the affective lessons modeled by faculty demonstrating support, collaboration, and extraordinary generosity in addition to expertise.

\section{CONCLUSION}

The ORL Emergencies Boot Camp helps jump start residents' skills at the beginning of their careers. The value of this novel educational approach is demonstrated by the fact that this course is currently being adopted, openly and with our collaboration, by multiple other faculty at a variety of institutions. We anticipate that regional implementation of similar programs will be the most feasible approach to expanding the availability of this type of learning opportunity, and that aggregation of information derived from these programs will be an important component of improving our educational strategies in the future.

\section{BIBLIOGRAPHY}

1. Falcone RA, Daugherty M, Schweer L, Patterson M, Brown RL, Garcia VF. Multidisciplinary pediatric trauma team training using high-fidelity trauma simulation. J Pediatr Surg 2008;43:1065-1071.

2. Allan CK, Thiagarajan RR, Beke D, et al. Simulation-based training delivered directly to the pediatric cardiac intensive care unit engenders preparedness, comfort, and decreased anxiety among multidisciplinary resuscitation teams. J Thorac Cardiovasc Surg 2010;140:646-652.

3. Anderson M, Leflore J. Playing it safe: simulated team training in the OR. AORN J 2008;87:772-779. 